QNueva Etinexaxia Revista Digital de OFilosofia ISSN 1850-3578 2013 - Vol. 8 - Número VIII - Resistencia, Chaco, Argentina. Pp. 21 - 38

\title{
La renuncia de Niels Bohr a la cosa en sí
}

Matías Oroño

Universidad de Buenos Aires. CONICET (Argentina)

Recibido: 05/04/2013

Aceptado: $27 / 05 / 2013$

\section{Resumen:}

El objetivo principal de este trabajo consiste en reflexionar acerca de los supuestos filosóficos que subyacen a la interpretación que Bohr efectúa acerca de la mecánica cuántica. Se señala que, en última instancia, Bohr renuncia a conocer lo que las cosas son en sí mismas, retomando de ese modo el espíritu de la revolución instaurada por Kant en la historia del pensamiento occidental. En primer lugar, me ocuparé de reconstruir el argumento presente en lo que se conoce como E.P.R. En este texto es cuestionada la completitud de la mecánica cuántica. En segundo término se analizara la respuesta brindada por Bohr. Y por último, me detendré en algunas cuestiones acerca de la relación entre Kant y Bohr.

Palabras clave: mecánica cuántica - Niels Bohr - Kant - cosa en sí - ontología

\begin{abstract}
:
The main objective of this paper is to reflect on the philosophical assumptions underlying the interpretation made by Bohr about quantum mechanics. It is noted that, ultimately, Bohr resigns to know what things are in themselves, thus returning to the spirit of the revolution introduced by Kant in the history of Western thought. First, I will reconstruct the argument present in what is known as E.P.R. In this text is questioned the completeness of quantum mechanics. Secondly, we analyze the response given by Bohr. And finally, I will consider some questions about the relationship between Kant and Bohr.
\end{abstract}

Keywords: quantum mechanics - Niels Bohr - Kant - thing in itself - ontology 
eNweva Etinesania Revista Digital de OFilosofia ISSN 1850-3578 2013 - Vol. 8 - Número VIII - Resistencia, Chaco, Argentina. Pp. 21 - 38

"Jamás debemos olvidar que "realidad" también es una palabra humana al igual que "onda" o "conciencia". Nuestra tarea es aprender a usar estas palabras correctamente - es decir, de manera desambigua y consistente." Niels Bohr ${ }^{1}$

\section{Introducción.}

Los desarrollos medulares de la mecánica cuántica han puesto en tela de juicio ideas filosóficas tradicionales vinculadas, por ejemplo, a la acción continua de la causalidad o la creencia en un mundo con ciertas características que subsisten con independencia a la intervención del sujeto que intenta conocerlo. Una de las figuras paradigmáticas pertenecientes al campo de la mecánica cuántica es sin lugar a dudas el neokantiano Niels Bohr. La polémica que intentaré analizar en el presente trabajo se remonta a mediados de los años 30 del siglo XX y tiene como contrincantes, por un lado, al ya mencionado Bohr como uno de los defensores de cierta interpretación acerca del valor de la mecánica cuántica y, por otro, a Albert Einstein, quien en aquel entonces ya había manifestado sus reservas acerca de las tesis sostenidas en el marco de la mecánica cuántica, la cual renunciaba entre otras cosas a la posibilidad de conocer lo que las cosas son con independencia del sujeto que experimenta y a las idea clásica de acción causal continua. Quizás, el trasfondo filosófico de esta disputa quede establecido con gran claridad si tomamos en cuenta las palabras del propio Einstein quien decía, refiriéndose a la mecánica cuántica, en un artículo publicado en el año 1936: "creer esto es lógicamente posible sin contradicción, pero es muy contrario a mi instinto científico que no puede renunciar a la búsqueda de una concepción más completa"².

El objetivo general de este trabajo consiste en reflexionar acerca de los supuestos filosóficos que subyacen a la interpretación bohriana de la mecánica cuántica, señalando que, en última instancia, Bohr renuncia a conocer lo que las cosas son en sí mismas, retomando de ese modo el espíritu de la revolución instaurada por Kant en la historia del pensamiento occidental. Se

1 Citado en: Wheeler, John Archibald and Zureck, Wohjciech Hubert, Quantum Theory and Measurement, Edited by John Archibald Wheeler and Wohjciech Hubert Zureck, Princeton, Princeton University Press, 1983. p. 5.

${ }^{2}$ Citado por Niels Bohr. Véase: Bohr, N., "Discussion with Einstein on epistemological problems in atomic physics". En: Quantum Theory and Measurement, Edited by John Archibald Wheeler and Wohjciech Hubert Zureck, Princeton, Princeton University Press, 1983. p. 43. En los casos en que no se indica el nombre del traductor, se trata de una traducción propia. 
Q Nueva Etinexaxio Revista Digital de OFilosofia ISSN 1850-3578 2013 - Vol. 8 - Número VIII - Resistencia, Chaco, Argentina. Pp. 21 - 38

tomará como hilo conductor la respuesta que el mismo Bohr ofrece al artículo conocido como E.P.R., el cual habría sido escrito por Einstein, Podolsky y Rosen. En primer lugar, me ocuparé de reconstruir el argumento presente en lo que se conoce como E.P.R, mediante el cual se cuestiona la completitud de la mecánica cuántica. En segundo término analizaré la respuesta de Bohr. Y por último, me detendré en algunas cuestiones acerca del kantismo que subyace a la postura de Niels Bohr.

\section{EPR y la supuesta incompletitud de la mecánica cuántica.}

En un artículo titulado "Can quantum-mechanical description of physical reality be considered complete" (1935) ${ }^{3}$, también conocido como "E.P.R.", desarrollan Albert Einstein, Boris Podolsky y Nathan Rosen una crítica a ciertos aspectos medulares de la mecánica cuántica ${ }^{4}$. A continuación, me encargaré de reconstruir el movimiento argumentativo presente en el mencionado artículo.

Los autores comienzan señalando que para evaluar el éxito de una teoría debemos formular dos preguntas, a saber: 1) ¿Es correcta la teoría?; 2) ¿Es completa la descripción dada por la teoría? En cuanto a la primera cuestión, queda establecido que la corrección de una teoría debe ser juzgada mediante el acuerdo entre las conclusiones de dicha teoría y la experiencia humana -la cual, en el caso particular de la física, adopta la forma de experimentos y mediciones-. Sin embargo, en el artículo que estamos analizando, los autores señalan que se ocuparán de la mecánica cuántica tomando como hilo conductor la segunda pregunta. Es decir, se cuestionará la completitud de la teoría y se supondrá que la misma es correcta. En este contexto, suponer que la mecánica cuántica es correcta significa asumir que existe un grado aceptable de concordancia entre las conclusiones de la teoría y los resultados que arrojan los experimentos y las mediciones.

\footnotetext{
${ }^{3}$ Einstein, A., Podolsky B., Rosen N., "Can quantum-mechanical description of physical reality be considered complete?". En: Quantum Theory and Measurement, Edited by John Archibald Wheeler and Wohjciech Hubert Zureck, Princeton, Princeton University Press, 1983. pp. 138-141.

${ }^{4}$ En sus comienzos Einstein fue muy entusiasta con el desarrollo de la mecánica cuántica, pero hacia el año 1935, este entusiasmo ya había comenzado a decaer. Lo que Einstein cuestionaba de esta teoría podría resumirse en dos puntos. El primero de ellos se refiere a la renuncia de la mecánica cuántica a conocer lo que las cosas son en sí mismas, con independencia de las observaciones o mediciones. El segundo punto, que molestaba a Einstein, se vincula al carácter probabilístico -por principio- de la mecánica cuántica, es decir, un tipo de probabilidad que podríamos llamar "ontológica", en la medida en que no se debe a falta de conocimientos. Véase: Fine, Arthur, "The Einstein-Podolsky-Rosen Argument in Quantum Theory", [entrada], Fecha de creación: 10 de mayo de 2004, fecha de actualización: 5 de agosto de 2009, [citado el 4 de abril de 2013]. URL = <http://plato.stanford.edu/archives/win2011/entries/qt-epr/>.
} 
Q Nueva Etinexaxio Revista Digital de OFilosofia ISSN 1850-3578 2013 - Vol. 8 - Número VIII - Resistencia, Chaco, Argentina. Pp. 21 - 38

Ahora bien, tal como lo formulan nuestros autores, el siguiente requisito debe ser cumplido por una teoría que aspire a la completitud: "todo elemento de la realidad física debe tener una contraparte en la teoría física" ${ }^{5}$. Como vemos, este requerimiento supone la distinción entre la teoría y la realidad e implica lo siguiente: basta con que encontremos un elemento de la realidad física que no tenga su contraparte en la teoría física para que esta última sea evaluada como incompleta. Una vez establecido este criterio, solo resta saber cuáles son los elementos de la realidad física de los cuales la teoría debería poder dar cuenta. Con el objetivo de satisfacer esta necesidad es introducido el siguiente criterio:

$\mathrm{Si}$, sin de ningún modo perturbar un sistema, podemos predecir con certeza (es decir, con probabilidad igual a uno) el valor de una cantidad física, entonces existe un elemento de realidad física correspondiente a esta cantidad física. ${ }^{6}$

Nos hallamos con un enunciado que enuncia bajo qué condiciones podemos afirmar que existe un elemento de realidad física. Estas condiciones son las siguientes:

1) no debemos perturbar de ningún modo el sistema dentro del cual se halla el posible elemento de realidad física.

2) debe ser posible predecir con certeza (con probabilidad igual a 1) el valor de una cantidad física.

Solo si se cumplen estas dos condiciones podemos afirmar que existe un elemento de realidad física, el cual se corresponde con el valor de la cantidad física que habríamos predicho con certeza.

El siguiente punto que nos interesa señalar en E.P.R es el siguiente: uno de los principios formulados en el desarrollo de la mecánica cuántica es el llamado "Principio de Indeterminación"7 . Este señala, a grandes rasgos, que el valor definido de la posición de una partícula no es predecible pero puede ser obtenido gracias a una medición directa. Ahora bien, tal medición perturba la partícula y altera su estado generando como consecuencia la siguiente situación: cuando la velocidad de una partícula es conocida, su posición es incognoscible por

\footnotetext{
${ }^{5}$ Op. cit. Einstein, A., y otros, "Can quantum-mechanical description of physical reality be considered complete?", p. 138.

6 Ibíd.

${ }^{7}$ Este habría sido formulado por Heisenberg. Véase: Heisenberg, W., "The physical content of quantum kinematics and mechanics". En: Quantum Theory and Measurement, Edited by John Archibald Wheeler and Wohjciech Hubert Zureck, Princeton, Princeton University Press, 1983. pp. 62-84.
} 


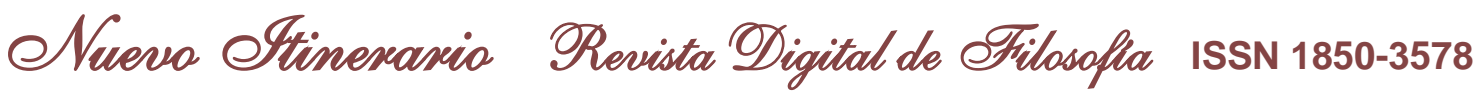
2013 - Vol. 8 - Número VIII - Resistencia, Chaco, Argentina. Pp. 21 - 38

principio; y a la inversa, cuando conocemos la posición de una partícula, el conocimiento de su velocidad queda excluido. Esto significa que en el marco de la mecánica cuántica, posición y velocidad no conmutan puesto que pertenecen a contextos diferentes. Dicho en otros términos, la mecánica cuántica muestra que:

[...] si los operadores correspondientes a dos cantidades físicas, supongamos $A$ y $B$, no conmutan, es decir, si $A B \neq B A$, entonces el conocimiento preciso de uno de ellos excluye el conocimiento del otro $^{8}$.

Los autores de E.P.R señalan que como consecuencia de estos resultados, la mecánica cuántica nos conduce al planteo de la siguiente disyuntiva: o bien la descripción de la realidad que nos ofrece la mecánica cuántica es incompleta, o bien cuando los operadores correspondientes a dos realidades físicas no conmutan, las dos cantidades no pueden tener realidad simultánea. En caso de predicar la verdad de la primera parte de esta disyunción, debemos aceptar la falsedad de la segunda parte; y a la inversa, si predicamos la falsedad de la segunda parte, debemos considerar como verdadera la primera. La estrategia argumentativa de Einstein, Podolsky y Rosen consistirá en mostrar que la segunda parte de la disyunción antes señalada es falsa, es decir, se mostrará que la proposición "cuando los operadores correspondientes a dos cantidades físicas no conmutan, las dos cantidades no pueden tener realidad simultánea" es falsa, lo cual es equivalente a sostener que la proposición "cuando los operadores correspondientes a dos realidades físicas no conmutan, las dos cantidades pueden tener realidad simultánea" es verdadera. Asimismo, tal demostración debería forzarnos a aceptar que la proposición "la descripción de la realidad que nos ofrece la mecánica cuántica es incompleta" es verdadera. El sentido de esta disyunción exclusiva supone que la llamada "función de onda" de la mecánica cuántica niega la posibilidad de aceptar la realidad simultánea de cantidades físicas cuyos operadores no conmutan. Así pues, si hallamos al menos un caso en el cual sea posible determinar la realidad simultánea de cantidades físicas cuyos operadores no conmutan, entonces la descripción de la realidad que nos ofrece la función de onda es incompleta.

\footnotetext{
${ }^{8}$ Op. cit. Einstein, A., y otros, "Can quantum-mechanical description of physical reality be considered complete?", p. 139.
} 
Q Nueva Etinexaxio Revista Digital de OFilosofia ISSN 1850-3578 2013 - Vol. 8 - Número VIII - Resistencia, Chaco, Argentina. Pp. 21 - 38

Veamos pues los lineamientos centrales del argumento propuesto por los autores de E.P.R para demostrar que es posible determinar la realidad simultánea de cantidades físicas cuyos operadores no conmutan:

1- supongamos que tenemos dos sistemas (a los cuales llamaremos "sistema 1" y "sistema 2") que interactúan entre los tiempos $\mathrm{t}=0$ y $\mathrm{t}=\mathrm{T}$, luego de dicho período no existe interacción alguna entre ambos sistemas.

2- conocemos los estados que estos sistemas poseen antes de la interacción.

3- dentro del período de interacción, podemos calcular con ayuda de la ecuación de Schrödinger los estados subsiguientes del sistema compuesto por el sistema 1 y el sistema 2.

4- según la mecánica cuántica solo podemos calcular el estado en el cual es dejado uno de los dos sistemas luego de la interacción por medio de nuevas mediciones.

5- el experimento de E.P.R muestra un caso en el cual es posible, por ejemplo, la siguiente situación: a partir de la medición del sistema 1 estamos en condiciones de predecir con certeza y sin perturbar de ningún modo el sistema 2, la posición y/o la velocidad del sistema 2. (Del mismo modo, podría predecirse el valor del sistema 1 partiendo de mediciones efectuadas sobre el sistema 2).

6- Del punto anterior se sigue que podemos determinar la posición y velocidad de un sistema sin necesidad de perturbarlo.

Conclusión: dado que hemos encontrado un caso en el cual es posible determinar la posición y velocidad de un sistema, debemos concluir que la descripción de la realidad que nos ofrece la mecánica cuántica es incompleta.

\section{La respuesta de Bohr a la crítica formulada en E.P.R}

Rosenfeld señala en un breve comentario publicado en 1967 que:

A pesar de su aparente claridad, el criterio en cuestión contiene de hecho una ambigüedad esencial encubierta en la aparentemente inocua restricción "sin perturbar el sistema" ${ }^{\prime \prime}$.

\footnotetext{
9 Rosenfeld, L., “Bohr's Reply. Commentary of Rosenfeld (1967)". En: Quantum Theory and Measurement, Edited by John Archibald Wheeler and Wohjciech Hubert Zureck, Princeton, Princeton University Press, 1983, p.142
} 
Bohr mismo señala en una breve artículo publicado en 1935 que "[...] el procedimiento de medición tiene una influencia esencial sobre las condiciones en las cuales reposa la mera definición de las cantidades físicas en cuestión" ${ }^{\prime 10}$. Poco tiempo después publica Bohr una respuesta una tanto más detallada en la cual desarrolla principalmente dos tesis. La primera de ellas consiste en indicar la ambigüedad esencial implícita en el criterio de realidad física formulado por los autores de E.P.R. La segunda tesis podría resumirse en la idea según la cual la complementariedad permitiría satisfacer cualquier demanda racional de completitud hacia la mecánica cuántica.

En el artículo de Bohr es comentada, a modo de ilustración, la situación a la cual se ve enfrentada la mecánica cuántica al intentar medir la posición y la velocidad de una partícula que atraviesa una rendija en un diafragma. En este caso, la interacción entre el instrumento de medición (el diafragma) y el objeto de estudio (la partícula) imposibilita un conocimiento preciso y simultáneo de la posición y la velocidad de la partícula en cuestión. Ahora bien, tal como señala Bohr, la imposibilidad de un análisis detallado de las reacciones entre la partícula y el instrumento de medición no es una particularidad del procedimiento aquí descripto, sino que se trata de una propiedad esencial concerniente al estudio de todos los fenómenos estudiados por la mecánica cuántica. Es decir, diferentes arreglos experimentales permitirán conocer con precisión o bien la posición o bien la velocidad de una partícula. En un caso conocemos sólo la posición, pero la influencia del instrumento -o mejor dicho, del arreglo experimental- impide un conocimiento preciso acerca de la velocidad de la partícula estudiada. A la inversa, cuando conocemos la velocidad, el arreglo experimental que nos permite tal conocimiento, nos impide por el mismo motivo una determinación de la posición de la partícula.

Por estos motivos, sostiene Bohr que la expresión "sin de ningún modo perturbar un sistema" contiene una ambigüedad esencial cuando es aplicada a los fenómenos estudiados por la mecánica cuántica, puesto que en el campo de la teoría cuántica los arreglos experimentales siempre afectan al objeto de estudio y, de ese modo, excluyen la posibilidad de conocer de manera simultánea la posición y la velocidad de una partícula ${ }^{11}$. Tal como señala Fine ${ }^{12}$, Bohr

\footnotetext{
${ }^{10}$ Bohr, N. "Quantum mechanics and physical reality". En: Quantum Theory and Measurement, Edited by John Archibald Wheeler and Wohjciech Hubert Zureck, Princeton, Princeton University Press, 1983, p. 144.

11 Dicho en otros términos, E.P.R ofrecería condiciones suficientes para reconocer un elemento de realidad física, sin embargo, la objeción de Bohr a dicho criterio es que dichas condiciones no pueden
} 
Q Nueva Etinexaxio Revista Digital de OFilosofia ISSN 1850-3578 2013 - Vol. 8 - Número VIII - Resistencia, Chaco, Argentina. Pp. 21 - 38

reconoce que en el argumento de E.P.R no existe una "perturbación mecánica" sobre el "sistema 2" cuando medimos el "sistema 1", sin embargo, Bohr estaría señalando la inevitabilidad de que el arreglo experimental o medición sobre el "sistema 1" implique una influencia en el tipo de predicción que podamos efectuar acerca del "sistema 2". Por ejemplo, si elegimos medir la posición del "sistema 1" estaremos en condiciones de predecir con certeza la posición de "sistema 2" pero no así la "velocidad".

Mediante el concepto de complementariedad Bohr intenta señalar que la mecánica cuántica podría ofrecer una respuesta a las demandas de completitud ${ }^{13}$. La complementariedad nos señala que diferentes arreglos experimentales nos permiten conocer de manera desambigua o bien la posición o bien la velocidad de una partícula. Se trata de conocimientos que se excluyen recíprocamente pero al mismo tiempo se complementan al dar cuenta de diferentes aspectos de lo que supuestamente es una misma realidad. Es importante señalar que dentro de cada arreglo experimental nos hallamos dentro de un contexto o región que es equivalente a las descripciones efectuadas por la mecánica clásica. Es decir, cada arreglo experimental con sus correspondientes resultados nos permite alcanzar conocimientos como si estuviésemos en el marco de la mecánica clásica. El problema propio de la mecánica cuántica surge cuando se pretende considerar de manera simultánea o superpuesta diferentes arreglos experimentales o contextos, pues ellos se excluyen y contradicen entre sí.

\section{1. El kantismo de Bohr}

Es importante señalar los aspectos filosóficos que subyacen a la posición bohriana respecto de la mecánica cuántica. Para ello seguiré en este punto la interpretación ofrecida por H. Pringe ${ }^{14}$. Es ampliamente conocida la tesis kantiana según la cual:

ser cumplidas consistentemente en el campo de la mecánica cuántica. Cfr. Op. cit. Fine, A., "The Einstein-Podolsky-Rosen Argument in Quantum Theory", artículo en línea.

${ }_{12}$ Op. cit. Fine, A., "The Einstein-Podolsky-Rosen Argument in Quantum Theory", artículo en línea.

${ }^{13}$ Aerts señala que la mecánica cuántica es incompleta pero no por los motivos aludidos en E.P.R (es decir, por la supuesta incapacidad para predecir los valores de un sistema sin perturbarlo), sino por su incapacidad de describir en un sistema único dos sistemas separados. El presupuesto de E.P.R consiste es sostener que la mecánica cuántica debería poder dar una descripción simultánea (en un único sistema) de sistemas separados. Véase: Aerts, D., "The Missing elements of reality in the description of quantum mechanics of E.P.R paradox situation". En: Helvetica Physica Acta, Vol. 57, 1984, pp. 421-428.

Sin embargo, la aceptación misma del postulado cuántico excluye la posibilidad de que la mecánica cuántica cumpla con los requisitos de completitud exigidos por E.P.R.

${ }^{14}$ Pringe, H., "La filosofía trascendental y la interpretación de Bohr de la teoría cuántica". En: Scientiae Studia, v. 10, n. 1, 2012, p. 179-94. 
Pverea Etinexaxio Revista Digital de OFilosofla ISSN 1850-3578 2013 - Vol. 8 - Número VIII - Resistencia, Chaco, Argentina. Pp. 21 - 38

Hasta ahora se ha supuesto que todo nuestro conocimiento debía regirse por los objetos; pero todos los intentos de establecer, mediante conceptos, algo a priori sobre ellos, con lo que ensancharía nuestro conocimiento, quedaban anulados por esta suposición. Ensáyese, por eso, una vez, si acaso no avanzamos mejor, en los asuntos de la metafísica, si suponemos que los objetos deben regirse por nuestro conocimiento; lo que ya concuerda mejor con la buscada posibilidad de un conocimiento de ellos a priori que haya de establecer algo acerca de los objetos, antes que ellos nos sean dados. ${ }^{15}$

Estas ideas conducen a la conocida "revolución copernicana" según la cual "[...] conocemos $a$ priori de las cosas sólo aquello que nosotros mismos ponemos en ellas ${ }^{\prime 16}$. Ahora bien, en el marco de la filosofía trascendental kantiana aquello que conocemos a priori de los objetos constituye una condición que hace posible al objeto en tanto objeto. Así pues, sabemos que todo objeto se hallará en relaciones de causalidad con otros objetos y que éstos se presentan intuitivamente de forma espacio-temporal. Nos hallamos pues con condiciones que constituyen al objeto y permiten que lo distingamos de las meras percepciones ilusorias. Es decir, mediante la aplicación de estas condiciones podemos distinguir lo real de lo meramente fantaseado. Por otro lado, en el marco de la filosofía trascendental hallamos condiciones que reflejan la unidad sistemática del conocimiento y permiten unificar los múltiples conocimientos que hemos adquirido.

Ahora bien, según $\mathrm{H}$. Pringe el postulado cuántico es el fundamento de la interpretación bohriana de la mecánica cuántica. Tal postulado sostiene que todo proceso atómico se caracteriza por una discontinuidad esencial. Es decir, se niega la ley de continuidad de todo cambio, según la cual una cosa al cambiar recorre todos los posibles cambios que se hallan comprendidos entre un estado inicial y un estado final. Por ello, en los procesos cuánticos hallamos transiciones mínimas o discretas que no pueden ser reducidas a otras menores. Si trasladamos esta situación al marco de la filosofía kantiana hallamos un problema, puesto que según Kant la ley de continuidad resulta de la aplicación de la categoría de causalidad (la cual constituye una de las condiciones necesarias de todo objeto en tanto objeto). De allí se sigue, que si en la mecánica cuántica aceptamos el postulado cuántico debemos negar tanto la ley de continuidad así como la aplicación de la categoría de causalidad. En otros términos, los

\footnotetext{
${ }^{15}$ Kant, I. Crítica de la razón pura, traducción de Mario Caimi, Buenos Aires, Colihue, 2007. (KrV, B XVI). Como es habitual, se cita esta obra utilizando las siglas KrV, seguidas de las letras A o B, para aludir a la primera o segunda edición.

${ }^{16}$ Op. cit. Kant, I., Crítica de la razón pura, (KrV, B XVIII)
} 


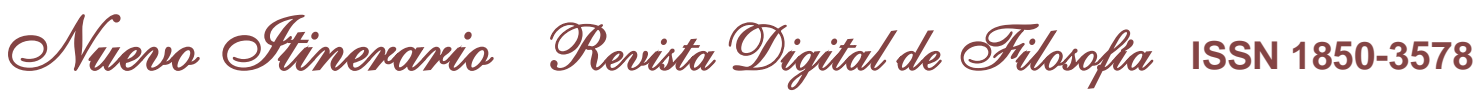
2013 - Vol. 8 - Número VIII - Resistencia, Chaco, Argentina. Pp. 21 - 38

procesos cuánticos parecen no poder constituirse como objetos de la experiencia posible, al menos en un sentido kantiano, y con ello, nos vemos incapaces de distinguirlos de las meras percepciones ilusorias. En Prolegómenos a toda metafísica del futuro Kant introduce la distinción entre "juicios de percepción” y "juicios de experiencia”. Mientras que los primeros son válidos de un modo meramente subjetivo, los últimos poseen una validez intersubjetiva que se debe a la aplicación de conceptos puros del entendimiento (entre ellos, el concepto puro de causalidad) gracias a los cuales constituimos la objetividad. Los siguientes son ejemplos mediante los cuales Kant intenta aclarar esta distinción:

Que el cuarto está caliente, que el azúcar es dulce, que el ajenjo es desagradable, son sólo juicios subjetivamente válidos. No exijo, en modo alguno, que, yo mismo, en todo momento, o cualquiera otro, haya de juzgarlo igual que yo; expresan solamente una relación de dos estados de sensibilidad en el mismo sujeto, a saber: en mí mismo y aun solamente en mi actual estado de percepción, y no deben, por consiguiente, tampoco, hacerse valer para el objeto; a éstos los llamo yo juicios de percepción. Otra cosa completamente distinta ocurre con los juicios de experiencia. Lo que me enseña la experiencia bajo ciertas circunstancias, me lo debe enseñar siempre y también a los demás, y la validez de lo mismo no se limita al sujeto o a su estado actual ${ }^{17}$.

Es decir, en el plano de la filosofía trascendental kantiana la aplicación de la categoría de causalidad permite constituir la objetividad superando de ese modo las percepciones que tienen un valor limitado al sujeto que juzga y al estado actual de la percepción. Gracias a la aplicación de las categorías podemos afirmar que lo que la experiencia nos enseña bajo ciertas circunstancias debe poder ser reproducido ya sea para mí mismo o para otros sujetos siempre que se respeten las circunstancias bajo las cuales la experiencia fue llevada a cabo. Es fácil comprender que en el campo de la mecánica cuántica debido a la aceptación del postulado cuántico ya no es posible aplicar la categoría de causalidad que nos permite constituir la objetividad mediante juicios de experiencia.

Asimismo, que no podamos distinguir lo objetivo en sentido kantiano de lo meramente subjetivo posee como contratara en el marco de la mecánica cuántica el hecho de que nos

\footnotetext{
${ }^{17}$ Kant, I., Prolegómenos a toda metafísica del futuro, traducción de Julián Besteiro, Losada, Buenos Aires, 2005, p. 83 (Ak. IV, 299). La aclaración entre paréntesis se refiere a la numeración establecida por la Edición Académica de los textos de Immanuel Kant.
} 
Q Nueva Etinexaxio Revista Digital de OFilosofia ISSN 1850-3578 2013 - Vol. 8 - Número VIII - Resistencia, Chaco, Argentina. Pp. 21 - 38

veamos incapacitados para distinguir el sistema cuántico del instrumento de medición utilizado. En suma, la negación de la acción causal continua genera una interacción tal entre el instrumento de medición y el sistema estudiado que nos vemos incapaces de atribuir realidad física independiente tanto al fenómeno observado como al instrumento de observación. Según Bohr, la interacción entre el instrumento de medición y el objeto supone la necesidad de renunciar a la idea clásica de causalidad.

Una primera diferencia que se desprende de lo expuesto hasta aquí es que en el marco de la física clásica podemos establecer el estado de un sistema aislado, dado que la continuidad de la acción causal permite:

[...] determinar el efecto del instrumento de medición sobre el sistema medido, para luego, sustrayendo tal efecto, calcular el estado del sistema más allá de su interacción con el instrumento de medición. Por el contrario, con la adopción del postulado cuántico, ese cálculo no puede ya llevarse a cabo. ${ }^{18}$

Que no se pueda calcular el estado del sistema aislado implica, por un lado, que la ley de acción causal continua no pueda ser aplicada, y por otro, supone que renunciemos a la posibilidad de observar el sistema en forma espacio-temporal. Es decir, si queremos definir el estado del sistema, debemos renunciar a todo tipo de perturbación externa, lo cual significa la imposibilidad de observarlo, y con ello, la renuncia a la representación espacio-temporal del sistema. Pero si queremos observarlo, debemos perturbarlo, con lo cual la definición del sistema se torna imposible. Es decir, podemos o bien observar el sistema o bien definirlo, pero no ambas cosas a la vez. Por el contrario, los sistemas clásicos se caracterizan por cumplir con ambos requisitos de manera simultánea.

Situados en este punto la pregunta que surge es la siguiente: ¿cómo podemos otorgar objetividad -en sentido kantiano- a los fenómenos cuánticos a fin de distinguirlos de la mera fantasía? A fin de responder esta pregunta debemos recordar que, según Bohr, un fenómeno cuántico no es más que el resultado de cierta situación experimental concreta. Dentro del marco otorgado por un arreglo experimental particular podemos y debemos manejarnos con el lenguaje clásico y dentro de dicho contexto la objetividad no representa un problema a ser resuelto. En cierto contexto, percibimos la onda como onda o la partícula como partícula. Es decir, si nos limitamos a las descripciones clásicas propias de cada situación experimental,

\footnotetext{
${ }^{18}$ Op. cit. Pringe, H., "La filosofía trascendental y la interpretación de Bohr de la teoría cuántica”, p. 182.
} 
Q Nueva Etinexaxio Revista Digital de OFilosofia ISSN 1850-3578 2013 - Vol. 8 - Número VIII - Resistencia, Chaco, Argentina. Pp. 21 - 38

tenemos garantizada la validez objetiva, pero ella sólo posee un carácter contextual, pues en la medida en que modifiquemos el arreglo experimental, la validez objetiva antes alcanzada se pierde y da lugar a una descripción clásica diferente - es decir, al cambiar el arreglo experimental se diluye la identidad del fenómeno cuántico para dar lugar a un objeto diferente-. En suma, sólo podemos otorgar objetividad a los fenómenos cuánticos si los consideramos como si fuesen fenómenos de la mecánica clásica, y por tanto, utilizando conceptos de objetos clásicos que nos permitan recuperar la causalidad y la espaciotemporalidad de los fenómenos experimentados. Cabe destacar que se trata de una estrategia que implica omitir la validez del postulado cuántico. Es decir, recuperamos la objetividad en un sentido contextual al precio de renunciar a lo más propio de los procesos cuánticos, a saber: la discontinuidad causal. Por tanto, debemos enfrentar la siguiente pregunta: ¿es posible recuperar la ontología cuántica luego de haberla considerado en términos de ontología clásica? El siguiente pasaje parece brindarnos el inicio de una posible respuesta:

[...] si bien cada fenómeno cuántico resulta una descripción espacio-temporal y causal, la multiplicidad de dichos fenómenos no puede ser unificada en una única imagen espacio-temporal y causal. Por el contrario, representaciones incompatibles (como, por ejemplo, imágenes de ondas e imágenes de partículas) parecen ser necesarias para una interpretación adecuada de los datos experimentales. $^{19}$

Es decir, la consideración completa de la evidencia experimental, y por tanto, de los diferentes contextos y arreglos experimentales será la clave que nos permitirá retornar al plano de la ontología propiamente cuántica. Es por ello que la complementariedad juega un rol tan importante en el marco de la interpretación bohriana de la mecánica cuántica, pues sólo gracias al carácter complementario de las descripciones clásicas contextuales que se excluyen entre sí, podemos decir que estamos hablando de una ontología cuántica. Dicho en términos de Bohr:

La evidencia obtenida bajo diferentes condiciones experimentales no puede ser abarcada en una única imagen, sino que debe ser considerada como

\footnotetext{
${ }^{19}$ Op. cit. Pringe, H., “La filosofía trascendental y la interpretación de Bohr de la teoría cuántica”, p. 186.
} 


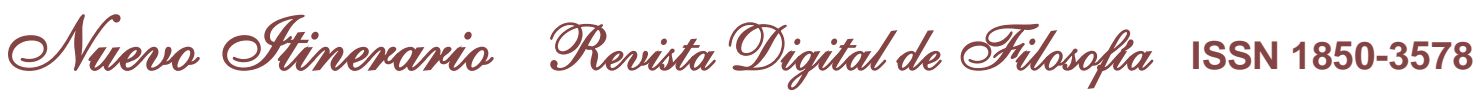
2013 - Vol. 8 - Número VIII - Resistencia, Chaco, Argentina. Pp. 21 - 38

complementaria, en el sentido de que sólo la totalidad de los fenómenos agota la información posible acerca de los objetos. (Bohr, 1949, p. 40)

Como un último paso en esta reconstrucción de la fundamentación kantiana de la mecánica cuántica ofrecida por Pringe, debemos señalar que será la noción de objeto cuántico la que nos permitirá unificar los diferentes fenómenos cuánticos propios de cada contexto particular. Dado cierto fenómeno cuántico podemos calcular mediante una ley probabilística el valor de todos y cada uno de los fenómenos del objeto cuántico. Es decir, el objeto cuántico unificará los fenómenos cuánticos a través de una ley probabilística llamada "función de onda" del sistema. Pringe señala que el objeto cuántico no es un "principio constitutivo" en sentido kantiano, pues cada fenómeno ya tiene garantizada su objetividad al estar descripto en términos clásicos. El objeto cuántico cumple lo que en términos kantianos llamaríamos "función regulativa", ya que es una condición que permite unificar sistemáticamente fenómenos cuya objetividad ya está garantizada. Cabe destacar que el concepto de objeto cuántico no puede ser exhibido directamente en la intuición por medio de esquemas (en sentido kantiano), sino que sólo puede ser exhibido en la intuición de manera indirecta a través de símbolos. Así pues, cada una de las descripciones clásicas será un símbolo del objeto cuántico, este último se comportará en algunos casos como si fuese una onda y en otros como si fuese una partícula.

3. 2. Siguiendo en este punto la investigación de $M$. Bitbol ${ }^{21}$, quisiera señalar que existen diversas maneras de fundamentar la mecánica cuántica a partir del marco ofrecido por la filosofía trascendental de Kant. Bitbol menciona cuatro interpretaciones kantianas al respecto: a) la interpretación de la información teórica, b) el realismo de la función de onda; c) el macrorealismo de Bohr y d) la lectura especulativa de Bohm.

Por cuestiones de extensión y dado que escapa a los objetivos de este trabajo no me detendré en el análisis de cada una de estas interpretaciones acerca de la mecánica cuántica. En lugar de ello, me centraré en el comentario de Bitbol acerca de la interpretación bohriana de la mecánica cuántica.

Tal como señala Bitbol, si bien Bohr nunca se expresó a estos términos, es posible distinguir una "micro-física" de una "macro-física". La micro-física consistiría en una suerte de

\footnotetext{
${ }^{20}$ Cit. en Pringe, H., "La filosofía trascendental y la interpretación de Bohr de la teoría cuántica", p. 188.

${ }^{21}$ Bitbol, M., "Reflective metaphysics: understanding quantum mechanics from a kantian standpoint". En Philosophica 83, 2010, pp. 53-83.
} 


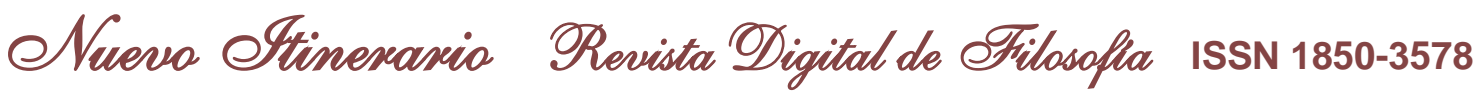
2013 - Vol. 8 - Número VIII - Resistencia, Chaco, Argentina. Pp. 21 - 38

simbolismo abstracto que permite predecir fenómenos experimentales cuyas condiciones experimentales están definidas por medio de conceptos macro-físicos. Es decir, la micro-física pareciera remitir a lo que Bohr denominaba objeto cuántico, mientras que la macro-física se refiere a los arreglos experimentales y a sus resultados descriptos a través de un lenguaje clásico. Según Bitbol, los conceptos macro-físicos son una condición de posibilidad del conocimiento intersubjetivo. Sin embargo, su validez sería sumamente restringida, pues se tratarían de condiciones de posibilidad antropocéntricas que posibilitarían las condiciones de posibilidad de la investigación micro-física. Dicho en términos de Bitbol:

[...] es una condición de posibilidad de segundo orden del conocimiento microfísico. Esta idea fue claramente formulada por Heisenberg: "Lo que Kant no ha anticipado es que estos conceptos a priori pueden ser condiciones para la ciencia y al mismo tiempo poseen un rango limitado de utilidad (Heisenberg, 1990, p. $78)^{22}$.

Es decir, los conceptos macro-físicos permiten descripciones clásicas, y con ello, fundamentan la posibilidad de referirnos a fenómenos espacio-temporales a los cuales podemos aplicar las categorías. Por un lado, dichos conceptos macro-físicos constituyen una condición de posibilidad directa para la física clásica. Por otro lado, son conceptos que indirectamente (y por ello en un segundo orden) posibilitan describir las condiciones experimentales mediante las cuales accedemos a la micro-física, es decir, al objeto cuántico propiamente dicho ${ }^{23}$. Probablemente, Heisenberg se refiere al limitado rango de utilidad de estos conceptos, dado que ellos sólo son útiles dentro del marco de una ontología clásica, en la cual la espaciotemporalidad y la causalidad rigen de manera simultánea. Ahora bien, en el marco de la mecánica cuántica esta limitación de los conceptos macro-físicos significa, además, que ellos sólo tienen validez dentro de determinado contexto o arreglo experimental, así pues, en un determinado contexto es útil el concepto de partícula, mientras que en otro contexto será apropiado el concepto de onda. Tan sólo quisiera aclarar contra esta observación, que Kant mismo ya era consciente del carácter antropocéntrico y limitado de lo que Bohr llamaría "descripciones clásicas", es decir, se trata de condiciones de posibilidad de la experiencia humana posible y, con ello, de la ciencia en relación a seres humanos que sólo pueden percibir

\footnotetext{
${ }^{22}$ Op. cit. Bitbol, M., "Reflective metaphysics: understanding quantum mechanics from a kantian standpoint", p. 67.

${ }^{23}$ Recordemos que este acceso al objeto cuántico posee un carácter "simbólico" y "contextual".
} 


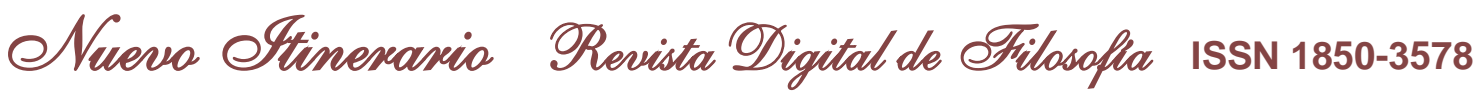
2013 - Vol. 8 - Número VIII - Resistencia, Chaco, Argentina. Pp. 21 - 38

los fenómenos a través de las formas sensibles del espacio y el tiempo y predicando de tales fenómenos ciertas categorías como la causalidad. Sin embargo, lo que parece ser ajeno al planteo kantiano es la multiplicidad de contextos y la falta de interconexión causal entre ellos. En este último sentido es cierto que la limitación contextual de los macro-conceptos es ajena al intento de unidad y universalización de la experiencia en sentido kantiano. Dicho en otros términos, la causalidad que presenta un mundo de ondas es diferente y discontinua respecto de la causalidad que se halla presente en un mundo de partículas. Es por ello que en la interpretación bohriana surge el concepto de complementariedad, pues de esa forma Bohr unifica los múltiples contextos, cada uno de los cuales está descripto mediante conceptos limitados a cierto contexto en particular. En rigor, el objeto cuántico no es experimentable (de modo espacio-temporal y causal) sino que es un postulado o un constructo simbólico que permite unificar diferentes arreglos experimentales que son incompatibles entre sí.

De estas reflexiones podemos extraer las siguientes conclusiones: en primer lugar, la interpretación de la mecánica cuántica efectuada por Bohr se halla mediada por la ontología clásica y, en particular, por el lenguaje de la física clásica, pues de esta manera podemos establecer las condiciones de posibilidad que nos permiten crear las situaciones experimentales desambiguas e intersubjetivas que se refieren de manera indirecta a un supuesto objeto cuántico:

\section{[...] por muy lejos que los fenómenos trasciendan el alcance de la explicación física clásica, el reporte de toda evidencia debe ser expresado en términos clásicos. El argumento es simplemente que por medio de la palabra "experimento" nos remitimos a una situación donde podemos decirle a otros lo que hemos hecho y qué hemos aprendido $[\ldots]^{24}$.}

En segundo lugar, el objeto cuántico o la supuesta ontología cuántica no puede ser objeto de conocimiento en sentido kantiano, sino que surge como un postulado necesario que permite unificar diferentes contextos experimentales. En suma, el aparato conceptual kantiano parece mostrar limitaciones a la hora de fundamentar la cognoscibilidad en sentido estricto de aquello a lo cual se refiere la mecánica cuántica y por ello será necesario apelar al artilugio según el cual poseemos un conocimiento indirecto o simbólico acerca del objeto cuántico. Quizás sea

\footnotetext{
${ }^{24}$ Bohr, N., "Discussion with Einstein on epistemological problems in atomic physics". En: Quantum Theory and Measurement, Edited by John Archibald Wheeler and Wohjciech Hubert Zureck, Princeton, Princeton University Press, 1983. pp. 9-49.
} 


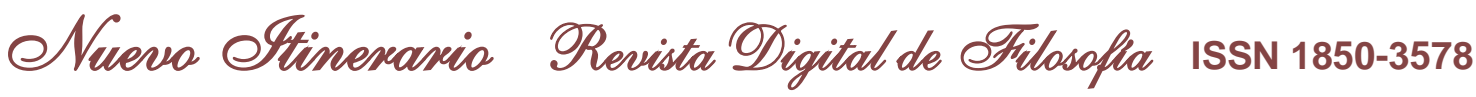
2013 - Vol. 8 - Número VIII - Resistencia, Chaco, Argentina. Pp. 21 - 38

necesario abandonar el sistema propiamente kantiano y pensar en desarrollos postkantianos para entonces fundamentar la cognoscibilidad directa del mundo cuántico, pues tal como Bitbol señala:

[...] se puede adoptar una definición pragmática del a priori en lugar de una puramente intelectual (Pihlström, 2003). De acuerdo con esta definición, una forma a priori no es más una condición intelectual necesaria de manera universal para el conocimiento objetivo, sino una condición pragmática local y provisionalmente necesaria para la determinación de cierto dominio de intervención experimental o tecnológica compartido intersubjetivamente. Tales formas pragmáticas a priori se corresponden íntimamente a la definición general de Reichenbach de un "principio constitutivo" cuya validez es relativa a cierto dominio de práctica (Reichenbach, 1965) ${ }^{25}$.

Es decir, si redefinimos los principios constitutivos ya no con pretensión de validez universal y necesaria, sino con una validez limitada a cierto dominio particular podríamos fundamentar la cognoscibilidad directa de la ontología cuántica ${ }^{26}$. Para ello deberíamos pensar al objeto cuántico ya no como un constructo simbólico al estilo bohriano, sino como un principio constitutivo valido únicamente en el campo de intervención de la mecánica cuántica. Claro esta que esta noción pragmática del principio constitutivo es ajena al pensamiento de Kant y corresponde a un desarrollo postkantiano. La objeción a esta interpretación que estamos elucubrando acerca del objeto cuántico como un a priori pragmático es la siguiente: ¿Es legítimo contar con tantos dominios de conocimiento como cantidad de a priori pragmáticos postulemos? ¿En qué sentido se puede hablar de conocimiento cuando somos incapaces de interconectar los diferentes dominios pragmáticos? Tendríamos un a priori para la mecánica cuántica, otro para la mecánica clásica, otro para la teoría de la relatividad, etc. Es decir,

\footnotetext{
${ }^{25}$ Op. cit. Bitbol, M., "Reflective metaphysics: understanding quantum mechanics from a kantian standpoint", p. 62.

${ }^{26}$ No queda claro en el pasaje recién citado si los a priori pragmáticos se limitan a cada uno de los contextos experimentales que hallamos dentro de la mecánica cuántica o si la mecánica cuántica en su totalidad posee un a priori pragmático que la diferencia de otros modos de hacer ciencia física. Personalmente, optaré por la hipótesis según la cual es posible pensar un a priori pragmático para la mecánica cuántica en su conjunto, lo cual no impide pensar distintos a priori pragmáticos propios de cada contexto experimental. A partir de esta lectura del a priori pragmático será posible pensar al objeto cuántico ya no como un postulado simbólico incognoscible de manera directa, sino como un "principio constitutivo" de conocimiento, el cual se halla limitado al campo de la mecánica cuántica.
} 


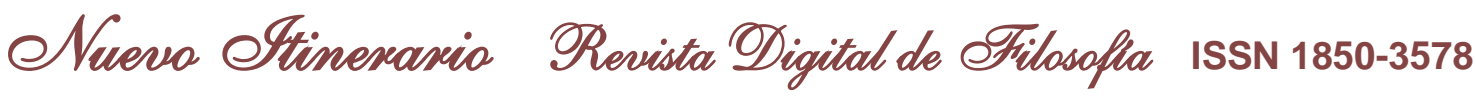
2013 - Vol. 8 - Número VIII - Resistencia, Chaco, Argentina. Pp. 21 - 38

resolveríamos pragmáticamente la unidad de la ontología cuántica al precio de aislarla de cualquier otro dominio científico ajeno al postulado cuántico.

\section{Conclusiones}

A todas luces, la discusión entre Bohr y Einstein que inició nuestro trabajo reposa sobre profundas diferencias de carácter metafísico. Aún si se tiene en cuenta que la posición bohriana puede ser analizada de distintas maneras ${ }^{27}$, podemos extraer algunas características acerca de su posición filosófica que deberían ser aceptadas con cierta unanimidad. Retomando una afirmación de Bohr que ya hemos citado en el presente trabajo, puede afirmarse que la idea según la cual "[...] el procedimiento de medición tiene una influencia esencial sobre las condiciones en las cuales reposa la mera definición de las cantidades físicas en cuestión"28 supone que en mecánica cuántica -al menos en sentido bohriano- los eventos carecen de autonomía. Esto significa que:

[...] todo evento es equivalente a una adscripción de valor observable y un observable sólo es definido en relación a una posibilidad instrumental efectiva de medirlo. En física cuántica, el contexto instrumental no es sólo la manera de acceder a un evento; es la forma de generarlo ${ }^{29}$.

Así pues, si aceptamos que en mecánica cuántica un evento se genera al ser medido, debemos aceptar el carácter contextual y completo de la teoría, pues la misma depende del contexto determinado por un arreglo experimental específico dentro del cual se sitúa el observador. La incompletitud sólo surgiría si se pretendiera tener acceso a elementos que se hallan fuera de la observación y medición que son posibles dentro de un contexto específico. Además, dado el principio de complementariedad, debemos tener en cuenta los diferentes contextos como descripciones complementarias que dan cuenta del objeto cuántico. De ese modo, quedaría garantizada la completitud dentro de cada contexto y posteriormente, al considerar el carácter

\footnotetext{
${ }^{27}$ Por ejemplo, hemos señalado que el objeto cuántico o bien podría ser interpretado como un principio regulativo que unifica contextos incompatibles cuya objetividad ya está garantizada, o bien podría ser pensado como un a priori pragmático que cumple un rol constitutivo -y no meramente regulativoaunque limitado al dominio particular de intervención propio de la mecánica cuántica (esta última es una hipótesis propia inspirada en la posición de Reichenbach -señalada por Bitbol-). No es mi intención atribuir esta posición al propio Reichenbach, pues para ello deberíamos tener un acceso a la obra del propio autor.

${ }^{28}$ Op. cit. Bohr, N., "Quantum mechanics and physical reality", p. 144.

${ }^{29}$ Op. cit. Bitbol, M., "Reflective metaphysics: understanding quantum mechanics from a kantian standpoint", p. 78.
} 
eNueva Etinexaxio Revista Digital de Prilosofia ISSN 1850-3578 2013 - Vol. 8 - Número VIII - Resistencia, Chaco, Argentina. Pp. 21 - 38

complementario de las descripciones mutuamente excluyentes quedaría garantizada la completitud de la teoría en un orden superior y más abarcativo que el contemplado por cada contexto particular. Sólo debemos recordar que cada contexto debe ser experimentado a través de cierto contexto instrumental -es decir, no podemos predecir el valor de un elemento sin perturbar el sistema al cual dicho elemento pertenece-. Por el contrario, la incompletitud que Einstein predica de la mecánica cuántica supone una ontología de eventos, propiedades y estados independientes a la observación y medición. Parafraseando las ideas de Bitbol, podemos señalar que en mecánica cuántica las paradojas desaparecen sólo si modificamos nuestras pretensiones cognoscitivas, pues ya no tenemos un acceso a lo que las cosas son en sí mismas con independencia de nuestra interacción con ellas, sino que debemos conformarnos con un entendimiento de las mismas relativo a nuestra situación como sujetos inmersos en cada contexto. En este sentido resultan apropiadas las palabras del propio Bohr quien afirma que "[...] una realidad independiente en el sentido físico ordinario no puede ser atribuida ni a los fenómenos ni a los instrumentos de observación” ${ }^{30}$.

\footnotetext{
${ }^{30}$ Bohr, N., "The quantum postulate and the recent development of atomic theory". En: Quantum Theory and Measurement, Edited by John Archibald Wheeler and Wohjciech Hubert Zureck, Princeton, Princeton University Press, 1983. p. 89.
} 